\title{
Rare cerebral vein thrombosis and COVID-19 vaccine: the role of orbit ultrasound
}

\author{
Piergiorgio Lochner $^{1}\left[\right.$ [ Mathias Fousse ${ }^{1} \cdot$ Joao Reinoldo Goi Junior ${ }^{1} \cdot$ Shrey Mathur $^{1} \cdot$ Fatma Merzou $^{1}$
}

Received: 10 September 2021 / Accepted: 17 December 2021 / Published online: 27 January 2022

(c) Fondazione Società Italiana di Neurologia 2021

To the Editor,

Vaccine-induced immune thrombotic thrombocytopenia (VITT) and cerebral venous sinus thrombosis (CVST) after viral vector vaccines (ChAdOx1 nCoV-19 vaccine, Ad26. COV2 vaccine) are a rare occurrence for which there is an emerging evidence base [1]. Here, we present a case of CVST without VITT after vaccination with ChAdOx1 nCoV-19 vaccine (AstraZeneca) and discuss the non-invasive diagnostics used to confirm the case.

A 20-year-old woman was admitted with severe holocephalic headache. Her symptoms started 10 days after having received the first dose of the ChAdOx1 nCoV-19 vaccine (AstraZeneca). Due to her headache, she stopped taking oral contraceptive. It should be noted that she had suffered from pulmonary embolism after patella surgery earlier, which was treated with rivaroxaban for 1 year.

No history of smoking was present.

Physical and neurological examination was normal. Initial laboratory investigations, including complete blood count, fibrinogen, screening panel for vasculitis, and collagenoses as well as extensive laboratory work-up for thrombophilia including protein $\mathrm{C}$, protein $\mathrm{S}$ antithrombin, activated protein $\mathrm{C}$ resistance, factor $\mathrm{V}$ Leiden, prothrombin gene mutation, homocysteine, lupus anticoagulant, anti-cardiolipin antibody, and beta 2 glycoprotein antibody, were normal.

Corona SARS-coronavirus-2 PCR (COBAS) nasopharyngeal swab was unremarkable. The patient had however slightly elevated D-dimer $(1.79 \mathrm{mg} / \mathrm{l})$.

Due to the continuing headache, which was not responsive to medication, and the history of positive cerebrovascular event as well as the slightly increase of D-Dimer,

Piergiorgio Lochner

piergiorgio.lochner@gmail.com

1 Department of Neurology, Saarland University Medical Center, University of the Saarland, 66421 Homburg, Germany we opted for further diagnostic work-up. First at all, we performed a neurological point-of-care of care ultrasound (Neuro-POCUS) using transorbital ultrasound. This demonstrated papilledema (Fig. 1C) which is a finding compatible with probable increased intracranial pressure (ICP) [2]. Subsequent ophthalmology review excluded ocular causes for bilateral disc swelling. The manual perimetry was normal. Fundus photography confirmed the papilledema (Fig. 2) [3].

Finally MR imaging of the brain showed thrombosis of two cortical veins without evidence of hemorrhage and infectious venous sinus thrombosis (Fig. 1A, B). The patient was diagnosed to have an extensive cerebral venous thrombosis on the basis of MRA findings.

Additional laboratory investigations revealed no evidence of thrombocytopenia. The results of the HIT panel including heparin-induced platelet activation (HIPA) test and PIPA test were negative. Other repeated laboratory testing did not demonstrate any thrombocytopenia. She was treated immediately with anticoagulant with enoxaparin 100 IE/ $\mathrm{kg}$ two times per day subcutaneously. The patient remained clinically stable. The headache resolved fully after several days of treatment. The patient was discharged 10 days after admission free of any symptoms. A follow-up examination 1 month after discharge was unremarkable. Follow-up fundoscopy and TOS showed no evidence of papilledema on either side.

We highlight this case, firstly, because the clinical diagnosis of cerebral vein thrombosis is sometimes challenging. Secondly, this case demonstrates the utility of NeuroPOCUS transorbital sonography as a non-invasive and repeatable bedside technique for the assessment of increased ICP. Thirdly, this case highlights the importance of safety monitoring as part of the vaccination strategy against SARSCoV-2. Particular attention should be paid to patients with a history of thrombotic events and taking the contraceptive pill. Further vigilance is required to identify clues to determine whether in some rare cases an immune-mediated reaction could be related or aggravated to the vaccine with 


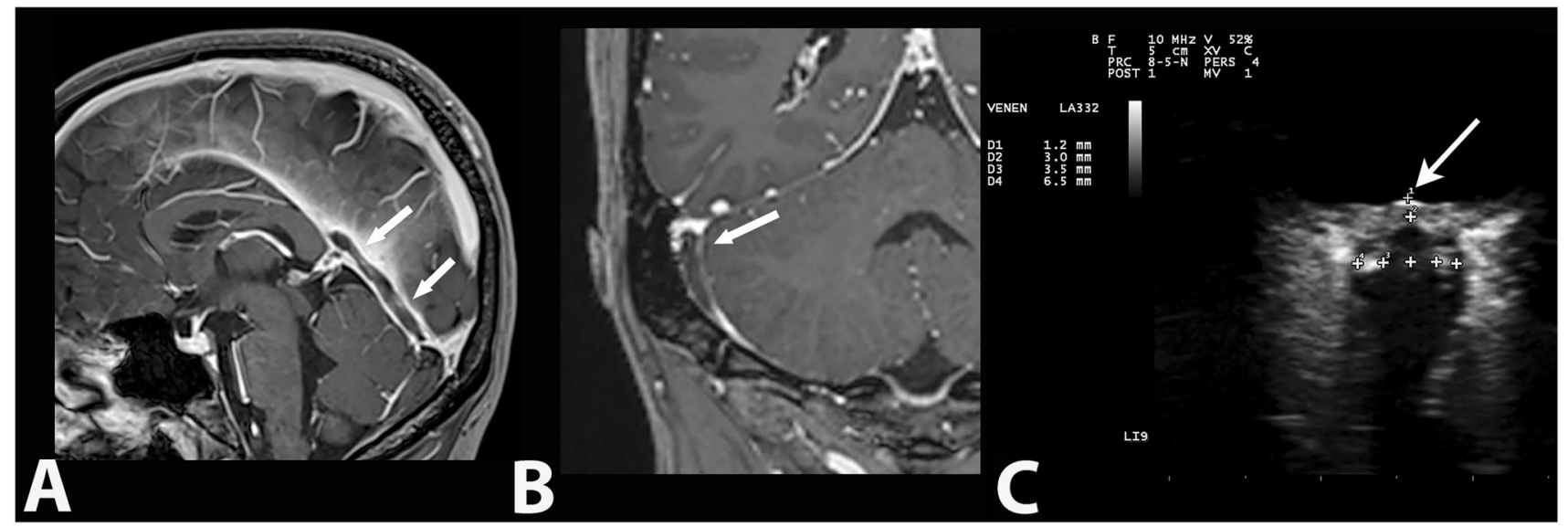

Fig. 1 Magnetic resonance angiography (MRI), T1-weighted sequence postcontrast showing the absence of flow in the deep venous cerebral system: MP-RAGE (A) sagittal and (B) coronal views. Transorbital sonography (TOS) depicting the optic disc eleva- tion (ODE). ODE is measured between the fundus and the dome of the papilla with the first caliper on the uppermost part of the swollen disc and the second caliper on the strongly reflecting line. Optic disc bulging $(\mathbf{C}$, arrow)

Fig. 2 Fundus photography eye: Modified Frisén scale for grading papilledema: grade 2 in the left papilla (L) and grade 1 in the right papilla $(\mathbf{R})$

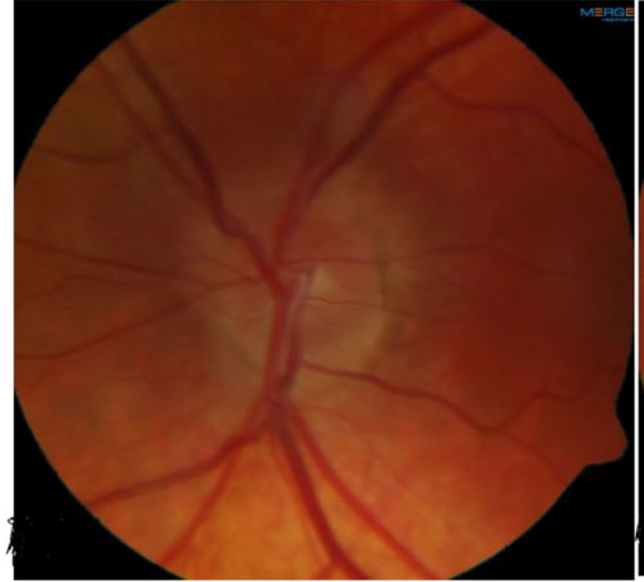

$\mathbf{L}$

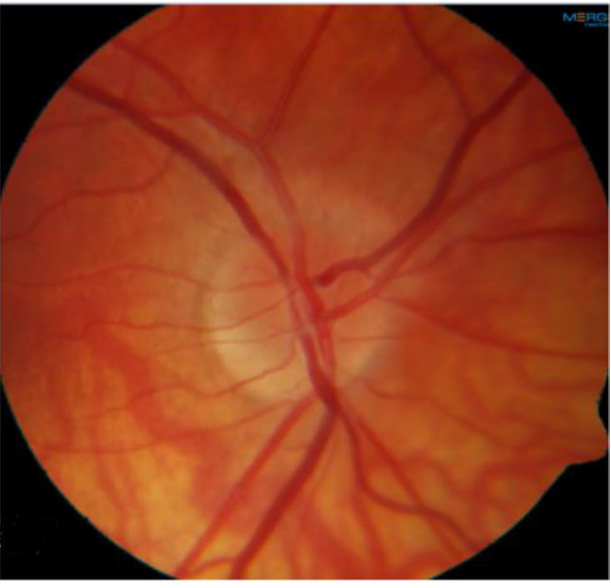

$\mathbf{R}$ or without vaccine-induced immune thrombotic thrombocytopenia, especially because of the temporal relationship with the vaccine and a history suggestive of pulmonary emboly in the past $[4,5]$. Given the chronological relationship of disease with COVID-19 vaccination, we believe that the use of oral contraceptive pills and vaccination have contributed to the cerebral venous sinus thrombosis [4].

\section{Declarations}

\section{Ethical approval Not applicable}

Consent for publication The patients have given written consent for publication in an academic journal.
Conflict of interest The authors declare that they have no competing interests.

\section{References}

1. Sharifian-Dorche M, Bahmanyar M, Sharifian-Dorche A, Mohammadi P, Nomovi M, Mowla A (2021) Vaccine-induced immune thrombotic thrombocytopenia and cerebral venous sinus thrombosis post COVID-19 vaccination; a systematic review. J Neurol Sci 428:117607

2. Lochner P, Czosnyka M, Naldi A, Lyros E, Pelosi P, Mathur S et al (2019) Optic nerve sheath diameter: present and future perspectives for neurologists and critical care physicians. Neurol Sci 40(12):2447-2457

3. Frisén L (1982) Swelling of the optic nerve head: a staging scheme. J Neurol Neurosurg Psychiatry 45(1):13-18 
4. Polack FP, Thomas SJ, Kitchin N, Absalon J, Gurtman A, Lockhart $S$ et al (2020) Safety and efficacy of the BNT162b2 mRNA covid-19 vaccine. N Engl J Med 383(27):2603-2615

5. Sánchez van Kammen M, Aguiar de Sousa D, Poli S, Cordonnier C, Heldner MR, van de Munckhof A et al (2021) Characteristics and outcomes of patients with cerebral venous sinus thrombosis in SARS-CoV-2 vaccine-induced immune thrombotic thrombocytopenia. JAMA neurology 78(11):1314-23
Publisher's note Springer Nature remains neutral with regard to jurisdictional claims in published maps and institutional affiliations. 